\title{
Optical computing: introduction by the guest editors to the feature in the 1 May 1988 issue
}

\author{
Ravindra A. Athale, Demetri Psaltis, and Kelvin Wagner
}

\begin{abstract}
The feature in the 1 May 1988 issue of Applied Optics includes a collection of papers originally presented at the 1987 Lake Tahoe Topical Meeting on Optical Computing. These papers emphasize digital optical computing systems, optical interconnects, and devices for optical computing, but analog optical processing is considered as well.
\end{abstract}

Optical computing systems are primarily considered for applications in which conventional computing technology is deemed inadequate because of massive computational requirements, or is inappropriate due to size, weight, or power constraints. This had led to the development of several diverse families of optical information processing systems. Analog optical signal processors are used in real-time on-board applications where size, weight, and power are at a premium, and absolute accuracy is not essential. The digital optical computer is intended to circumvent the speed limitations of conventional electronic computers by using high-speed nonlinear optical switches and speed of light communications without clock skew. Recently, two more families of optical processors have emerged. The goal of an optical interconnection system is to communicate between VLSI chips or computer systems, or to act as an optical routing network in a fiberoptic telecommunications system. Optical neural networks are based on the adaptive global interconnection of a large number of simple nonlinear threshold units, to create a robust dynamical system with interesting emergent computational capabilities, and are appropriate for implementation with imperfect optical hardware. To assist the dissemination of information and ideas in this multidisciplinary field, the Optical Society of America has organized a series of topical meetings on optical computing at Lake Tahoe, Nevada.

Ravindra Athale is with BDM Corporation, 7915 Jones Branch Drive, McLean, Virginia 22101; Demetri Psaltis is with California Institute of Technology, Department of Electrical Engineering, Pasadena, California 91125; and Kelvin Wagner is with University of Arizona, Optical Sciences Center, Tucson, Arizona 85721.

Received 5 March 1988.

0003-6935/88/091641-02\$02.00/0.

(C) 1988 Optical Society of America.
Some papers from the first meeting were collected in the issues of Applied Optics published 15 May 1986 and 15 June 1986, and some papers from the second meeting are collected in this 1 May 1988 issue.

The second Optical Society of America Topical Meeting on Optical Computing was held 16-18, March 1987 at Lake Tahoe, Nevada, in cooperation with the Society of Photo-Optical Instrumentation Engineers. This meeting was ttended by several hundred researchers in the fie tof optical computing devices and systems, and it br ught together workers in optical computing with those attending the photonic switching meeting held 18-20 March. The optical computing conference emphasized a diverse combination of research in algorithm and architecture development, systems and applications studies, and recent results in materials and device design. This issue of Applied Optics includes expanded versions of some of the papers presented at the topical meeting, along with some related papers. The focus of this meeting was on digital and nonlinear approaches to optical computing, with only a few papers exploring the more traditional analog optical signal processing approaches to optical information processing, and this balance is reflected in the papers collected in this issue. The papers are grouped into five topical areas: digital and residuebased optical computing, symbolic substitution approaches to digital optical computing, optical interconnection networks, optical devices and materials, and optical signal processing. Several of the papers fit into more than one category and have been grouped as deemed most appropriate by the guest editors.

Three different approaches to general-purpose digital optical computers are presented in the first three papers. They are all based on the detection of a dark condition at a detector or nonlinear optical gate, and all of these approaches use dual rail data representation, but the resulting architectures differ considerably. The first paper by Taboury, Wang, Chavel, 
Devos, and Garda presents the principles of shiftinvariant optical cellular processors, and contrasts cellular automatas with symbolic substitution and neural networks. The next paper by Murdocca, Huang, Jahns, and Streibl presents the philosophy and logic design of regularly interconnected perfect-shuffle circuits with uniform fan-in and fan-out of two, and optical addition is used as an example of a general programmable optical logic array. The paper by Guilfoyle and Wiley outlines the architectural development of digital optical computers that implement combinatorial logic using multichannel acoustooptic Bragg cells to input preprocessed vector binary data in a systolic fashion to an array of detectors acting as large fan-in and-or-invert gates.

The next two papers and a companion paper in the Letters section look at residue arithmetic approaches to optical numerical computations, thereby avoiding digital carry operations. The paper by Goutzoulis, Malarkey, Davies, Bradley, and Beaudet presents several designs for optical lookup tables to implement residue arithmetic, and a compact experimental prototype is demonstrated. The paper by Capps, Falk, and Houk uses a symbolic substitution approach to performing residue arithmetic. A related note in the letters section by Falk, Capps, and Houk presents the design and implementation of a crossbar-based optical residue processor.

The next group of papers explores the technique of symbolic substitution, originally developed by Alan Huang of AT\&T Bell Laboratories, as an approach to general-purpose digital optical computing. The first paper by Brenner discusses some of the limitations facing conventional computing, and a programmable optical symbolic substitution architecture is presented to circumvent these limitations. The following paper by Mait and Brenner presents details of the design of an optical symbolic substitution system that uses phase-only holograms for the optical interconnection elements. Cloonan examines the system efficiencies of three symbolic substitution architectures, sequential rule, parallel rule, and single rule based systems, all based on SEEDs and symmetric SEED flip-flops. Several possible applications of symbolic substitution operations in image processing of binary and multibit-plane images are explored by Goodman and Rhodes; this is an inherently parallel problem naturally amenable to parallel optical processing approaches. The final paper in this section by Wang et al. takes a practical look at the difficulties of an experimental implementation of symbolic substitution for simple pattern recognition and a related holographic associative memory for fingerprints, using thermally nonlinear interference filters as the decision-making-elements.

The optical interconnection networks section examines optical approaches to interconnection technologies. The first three papers examine various types of optical switching network, and then the limitations of . optical interconnections of VLSI and photorefractive neural networks are presented in the next two papers.
The first paper by Stirk and Athale gives several designs of optical compare-and-exchange modules for optical shuffle-exchange self-routing networks. The experimental implementation of an optical interconnection network based on electrically addressed, ferroelectric liquid crystal, halfwave plates and polarizing beam splitters is discussed by Johnson, Surette, and Shamir, and crosstalk mechanisms of this approach are determined. The paper by Lin, Krile, and Walkup presents the design and use of a 2-D optical Clos interconnection network, which is based on a hierarchy of smaller crossbars. A comparison between optical and electrical interconnects for VLSI chips and systems is reviewed in the paper by Feldman, Esener, Guest, and Lee and the operating regime for which the optical approach may be superior is delineated. The limitation on the number of interconnections that can be stored in a photorefractive crystal is examined in the paper by Psaltis, Brady, and Wagner, and architectures and experimental results of the optical implementation of learning algorithms using these crystals are presented.

The next group of papers examines both experimentally and theoretically a number of new devices with potential for use in optical computing systems. In the first paper, Cheng and Partovi experimentally investigate the index grating lifetimes in photorefractive GaAs, and they find a range of lifetimes suitable for several optical processing applications. A new thermally coupled, dual cavity, nonlinear Fabry-Perot etalon is presented by Craft, in which a low-power switching beam is able to switch a high-power cavity, modulating a high-power pump beam and resulting in a large fan-out operation. A different type of bistable device, demonstrated by Jager, Niessen, Forsmann, Thienpont, and Van Geen is based on a thermal selfelectrooptic effect in Cr-doped GaAs. A new type of three-port logic device, based on a diffusive nonlinear interface, is theoretically modeled by Cuykendall, and compared with optical Fredkin gates, and circuit topologies for optical adders using this multibeam switch are presented.

The final two papers might be classified as optical signal processing instead of optical computing since they are concerned with linear transformations of electrical signals. Integrated optics will undoubtably play an important role in the development of practical optical signal processing systems, and the paper by Zhang and Tsai presents an important step toward this goal, with the integration of a microlens array with a herringbone electrode array for the implementation of matrix multiplications. The final paper by Haney and Psaltis presents a system for the real-time imag. ing of spotlight-mode synthetic-aperture radar data using laser diode, acoustooptic, and charge-coupled device technologies, arguably the most mature device technologies for optical signal processing available today.

The guest editors would like to thank the reviewers for their invaluable assistance in producing this special issue. 